\title{
Perturbation-based balance assessment: Examining reactive balance control in older adults with mild cognitive impairments
}

\author{
LAKSHMI N KANNAN and TANVI S BHATT*
}

Department of Physical Therapy, The University of Illinois at Chicago, Chicago, Illinois, USA

Received: June 8, 2021 • Accepted: July 17, 2021

Published online: September 16, 2021

C 2021 Akadémiai Kiadó, Budapest

\begin{abstract}
Background: Older adults with mild cognitive impairment (OAwMCI) present subtle balance and gait deficits along with subjective memory decline. Although these presentations might not affect activities of daily living (ADLs), they attribute to a two-folded increase in falls. While changes occurring in volitional balance control during ADLs have been extensively examined among OAwMCI, reactive balance control, required to recover from external perturbations, has received little attention. Therefore, this study examined reactive balance control in OAwMCI compared to their healthy counterparts. Methods: Fifteen older adults with mild cognitive impairment (OAwMCI), fifteen cognitively intact older adults (CIOA) (>55 years), and fifteen young adults (18-30 years) were exposed to stance perturbations at three different intensities. Behavioral outcomes postural COM state stability, step length, step initiation, and step execution were computed. Results: Postural COM state stability was the lowest in OAwMCI compared to CIOA and young adults, and it deteriorated at higher perturbation intensities $(P<0.001)$. Step length was the lowest among OAwMCI and was significantly different from young adults $(P<0.001)$ but not from CIOA. Unlike OAwMCI, CIOA and young adults increased their step length at higher perturbation intensities $(P<0.001)$. OAwMCI showed longer recovery step initiation times and shorter execution times compared to CIOA and young adults at higher perturbation intensities $(P<0.001)$. Conclusion: OAwMCI exhibit exacerbated reactive instability and are unable to modulate their responses as the threat to balance control altered. Thus, they are at a significantly higher risk of falls than their healthy counterparts.
\end{abstract}

\section{KEYWORDS}

falls-risk, slip-perturbation, Alzheimer's related dementia (ADRD), compensatory stepping responses, scaling

* Corresponding author. Department of Physical Therapy, The University of Illinois at Chicago, 1919 W Taylor St, (M/C 898), 60612, Chicago, IL, USA. Tel.: +1 312355 4443; fax: +1 312996 4583. E-mail: tbhatt6@uic.edu 


\section{INTRODUCTION}

Mild cognitive impairment is a state of subjective memory decline but an absence of dementia with intact general intellectual function affecting about $15-20 \%$ of the older adults in the United States [1-3]. Although such a state may not interfere much with activities of daily living (ADLs), these older adults experience subtle balance and gait deficits contributing to the two-folded increase in falls when compared to their healthy counterparts $[4,5]$. Furthermore, consequences of these falls lead to poorer quality of life and long-term disability, giving rise to an increased risk of developing dementia or in some cases, Alzheimer's disease [6-8]. If efficacious fall prevention strategies among older adults with mild cognitive impairment (OAwMCI) are to be devised, understanding the underlying causative mechanisms of falls is essential.

Balance control is an important skill to prevent a fall for which the central nervous system (CNS) uses anticipatory postural adjustments against predicted balance threats via voluntary movements as well as reactive balance control against unpredicted balance threats via compensatory strategies $[9,10]$. Studies related to OAwMCI have extensively focused on assessing balance control under volitionally driven events (e.g., standing, obstacle crossing, getting up from a chair, walking) which are basic tasks needed to perform independent ADLs [11-13]. These studies report that such volitional-based performance was impaired (i.e., showed increased postural sway, reduced gait speed, increased step length variability) in OAwMCI compared to cognitively intact older adults (CIOA) [11-13]. Hence, training studies have also focused on improving balance control under volitionally driven events. On the other hand, reactive balance control, the primary defense mechanism against unpredicted balance threats, has not been extensively examined in OAwMCI.

Behavioral and neurophysiological studies have applied large intensity perturbations via computer-controlled equipment (platform or treadmill) to investigate reactive balance control among CIOA $[14,15]$. The existing literature pertained to the examination of reactive balance control in OAwMCI have used clinical tests such as the Balance Evaluation Systems Test (BESTest) [15]. Although it evaluates balance control on several domains, the manual therapistinduced perturbation to assess reactive balance control does not determine the causative mechanism of falls from biomechanical aspects. Furthermore, the therapist-induced perturbation is subjective, and the intensity of perturbation is considerably small compared to a real-life fall event. While falls among OAwMCI are far more precarious than CIOA, the influence of cognitive impairment on the ability to respond quickly and recover balance control, especially against unpredicted externally induced perturbations, remains unknown.

There are different compensatory strategies recruited by the CNS in response to unpredicted external perturbations. These include the in-place ankle or hip strategies in response to small perturbations as well as the change in support responses such as stepping or grasping in response to larger perturbations that serve to re-establish stability (i.e., its COM position and velocity within the displaced base of support) $[9,10]$. While evidence indicates that postural responses would be initiated by the short (spinal segmental) or long-loop (brain stem) reflexes, it is postulated that sensory information is simultaneously relayed to higher cortical centers to refine the response via the transcortical loop based on the internal model (or estimated) stability limits and the actual stability [16-18]. It is known that due to the age-associated decline in sensorimotor systems, this ability to perceive and integrate sensorimotor information to initiate stepping is delayed, resulting in impaired stepping responses and decreased reactive postural 
stability in CIOA compared to healthy young adults [19]. Studies report that compared to young adults, older adults, especially fallers, demonstrated an increased number of compensatory steps, delayed step initiation, and experienced limb collisions in response to large intensity perturbations $[14,15]$. This seems reasonable as there is a decline in age-associated factors such as muscle strength, endurance, flexibility, and reaction time, all of which are necessary for compensatory stepping $[20,21]$. Whether a state of subjective cognitive decline exacerbates reactive balance instability in response to large intensity perturbations remains unclear.

Apart from modifying the type of strategy, the CNS can further modify the magnitude of response based on the perturbation threat $[19,22]$. It can improve or maintain reactive stability by parametrizing (increasing or decreasing) muscle and joint forces based on the perturbation intensity perceived, an ability known as scaling [23, 19]. In a related manner, older adults demonstrated a tendency to initiate stepping at lower perturbation intensities, had decreased reactive stability without being able to scale their step length at higher perturbation intensity, and exhibited increased muscle co-contraction [19, 24]. These responses were more impaired among people with cortical lesions such as stroke [19]. There is enough evidence that OAwMCI also suffer certain cortical and subcortical structural damage such as decreased gray matter volume, loss of white matter integrity, cortical thinning, and hippocampal atrophy, which have been associated with increased risk of falling [25, 12, 26, 27]. However, whether such cortical and subcortical damage in OAwMCI may deteriorate reactive ability against large intensity perturbations needs to be explored.

For these reasons, this preliminary study aims to examine reactive balance control via standardized tests in OAwMCI compared to their healthy counterparts. Furthermore, this study aims to examine the ability to scale balance recovery responses when the perturbation magnitude increases among OAwMCI.

\section{METHODS}

\section{Participants}

The study included older adults above the age of 55 years with $(n=15)$ or without $(n=15)$ cognitive impairment and young adults $(n=15)$ between the age of 18-30 years after obtaining a written informed consent. Cognitively healthy older adults were included from a previous data set of which only partial data set was used (Institutional review board \#2009-0053) [19]. Older adults with mild cognitive impairment were recruited recently and no data related to this has been published (Institutional review board \#2018-1257). All the projects were approved by the University of Illinois at Chicago (UIC) institutional review board. Participants were recruited by posting flyers at the UIC College of Applied Health Sciences building, nearby independent living senior centers, bus stops, train stations, and grocery stores.

\section{Participants' eligibility}

The Montreal Cognitive Assessment (MoCA) scale was used to determine the cognitive status. Older adults with a score of more than 25 out of 30 points were considered cognitively intact and those with a score of less than 25 out of 30 points were considered as having a mild cognitive impairment [28]. Older adults with the presence of any neurological, cardiovascular, or 
musculoskeletal impairments as well as the inability to stand independently without an assistive device were excluded from the study, as such impairments may interfere with analysis and focus of the study. Heel bone density scan was measured for older adults using the Lunar Achilles Insight EXPII (General electric company, Milwaukee, Wisconsin, USA), and those with a Tscore of less than -2.0 were classified as osteopenia or osteoporotic and were excluded $[29,30]$.

\section{Reactive balance control test}

A stance perturbation test was administered using the Active step (Simbex, Lebanon, NH) motorized treadmill, and the full-body kinematics were recorded via Cortex software using an eight-camera motion capture system (Motion Analysis, Corporation, Santa Rosa, CA) with a sampling rate of $120 \mathrm{~Hz}$. An overhead metal arch with an attached safety harness secured the participants and prevented their knees to contact the belt surface in case of a fall. Helen Hayes marker set with 29 markers was placed bilaterally on bony landmarks to compute the center of mass (COM), and an additional marker was placed on the treadmill belt to identify the instant perturbation onset (i.e., sudden treadmill belt acceleration). A load cell was connected in series with the harness to measure the amount of body weight exerted on the harness. Participants attained a comfortable stance position with their feet shoulder-width apart. They were instructed to execute a natural response to regain their balance by taking a step upon a sudden forward movement of the belt (slip-like perturbation) (Fig. 1a). A familiarization trial was provided before the actual test and participants were unknown to perturbation onset. The participants were exposed to one perturbation each at three different perturbation intensities (displacement/ velocity/acceleration) which were randomized across the three groups (OAwMCI; CIOA; young adults). This was done to avoid adaptation to slip-perturbation and truly examine reactive responses at specific unpredictable perturbation intensities. For intensity I, the belt moved forward (slip-like) with a displacement of $0.2 \mathrm{~m}$ for $0.65 \mathrm{~s}$ at $0.31 \mathrm{~m} \mathrm{~s}^{-1}$ with an acceleration of $7.75 \mathrm{~m} \mathrm{~s}^{-2}$. For intensity II, the belt moved forward with a displacement of $0.2 \mathrm{~m}$ for $0.28 \mathrm{~s}$ at $0.67 \mathrm{~m} \mathrm{~s}^{-1}$ with an acceleration of $16.75 \mathrm{~m} \mathrm{~s}^{-2}$. For intensity III, the belt moved forward with a displacement of $0.3 \mathrm{~m}$ for $0.38 \mathrm{~s}$ at $0.86 \mathrm{~m} \mathrm{~s}^{-1}$ with an acceleration of $21.5 \mathrm{~m} \mathrm{~s}^{-2}$. Each perturbation trial was 10-15 s apart after the participant retained their original stance position. Kinematic variables, such as the postural COM state stability, step length, step initiation, and step execution, were computed using a custom-written algorithm in MATLAB version 2014b (The MathWorks Inc., Nactick, MA).

\section{Clinical measures}

All of the older adults with and without cognitive impairment were assessed using the Berg Balance Scale (BBS) to determine any standing balance impairments and their scores are reported in Table 1 [31].

\section{Outcomes}

Perturbation outcome. Each perturbation trial was identified as a fall or a recovery. A fall was identified when the force exerted on the load cell (i.e., participant supported by the harness) exceeded $30 \%$ of the total body weight for more than $1 \mathrm{~s}$ after the perturbation onset [32] or if the participant failed to initiate a compensatory stepping response resulting in a catch of the 
harness. Otherwise, the trial was identified as recovery with or without loss of balance. Recovery with no loss of balance was identified when the participants exhibited a feet in-place response. A backward recovery compensatory step was identified when the compensatory stepping leg heel landed posterior to the stance leg [19]. The total number of compensatory steps was noted for all participants.

Postural COM state Stability. Reactive postural COM state stability obtained post-perturbation onset was the primary variable of interest to examine differences in reactive balance control between groups as well as change across different intensities. The COM state stability was computed relative to the theoretical thresholds (dynamic feasibly stability boundary) for backward balance loss [33]. The stability value represents the shortest distance of the relative COM state (i.e., its position and velocity) to the theoretical boundary (Fig. 1c) [33]. The relative COM position was derived by expressing the absolute COM position relative to the rear edge of the base of support (BOS), specifically done by subtracting the COM position from the rear edge of the BOS. Similarly, the COM velocity was expressed relative to the velocity of the rear BOS. The COM position was normalized to each individual's foot length and COM velocity was normalized by the factor $\sqrt{ } g \times b h$, where $g$ is the gravitational acceleration and $b h$ is the individual's body height (Note $\times$ indicates multiplication) [34]. Thus, at instances such as liftoff of the compensatory step during a perturbed trial when the COM moves posterior to the BOS, values will be negative indicating greater instability. At the instance of touchdown of the compensatory step, the relationship between the COM and BOS is re-established and hence the $\mathrm{COM}$ is anterior to the BOS resulting in positive values.

Compensatory step characteristics. While both the temporal (step times) and spatial (step length) parameters were secondary variables to examine change in reactive balance control between groups, only step length was a secondary variable to examine change in magnitude of the response with change in perturbation intensity. This method was done as scaling ability is predominantly observed within spatial parameters and does not affect temporal characteristics as they both are differently affected by aging $[19,35]$.

The step parameters were computed as follows:

Step length. The anteroposterior displacement of the stepping limb's heel from perturbation onset to touchdown (TD) of the same limb for the first and second compensatory steps normalized to the individual's body height is defined as step length.

Step initiation. The time taken to lift the "heel off" of the stepping (recovery compensatory) limb after the perturbation onset is defined as step initiation and is measured in seconds.

Step execution. The time that is taken from lifting the "heel off" of the stepping (recovery compensatory) limb to "heel TD" of the stepping (recovery compensatory) limb after the perturbation onset is known as step execution and is measured in seconds.

\section{Statistical analysis}

Demographics and baseline clinical measures. One-way analysis of variance (ANOVA) was conducted to determine the demographic differences between the three groups (age, height, 
weight). Gender differences were determined using a chi-square test between the three groups. One-way ANOVA was conducted to determine differences in the BBS and MoCA test between OAwMCI and CIOA.

Perturbation outcome. Falls percentage was determined among each group at each perturbation intensity. $3 \times 3$ repeated-measures ANOVA was conducted to compare the group (OAwMCI vs CIOA vs young adults) differences at three different perturbation intensities (Intensity I, II, \& III) in number of compensatory steps taken. Post-hoc analysis with Bonferroni's correction was performed to resolve the main effects and interactions (Group $\times$ Intensity).

Reactive balance control test. For the first compensatory step, four $3 \times 3$ repeated measures ANOVA was conducted to compare the group (OAwMCI vs CIOA vs young adults) differences at three different perturbation intensities (Intensity I, II, \& III) in postural COM state stability, step length, step initiation, and step execution. Post-hoc analysis with Bonferroni's correction was performed to resolve the main effects and interactions (Group $\times$ Intensity). Furthermore, one-way ANOVA was conducted to determine differences within each group and each intensity in postural COM state stability, step length, step initiation, and step execution, and was followed up with a post-hoc Bonferroni's test to resolve main effects.

For the second compensatory step, four $3 \times 2$ repeated measures ANOVA was conducted to compare the group (OAwMCI vs CIOA) differences at three different perturbation intensities (Intensity I, II, \& III) in postural COM state stability, step length, step initiation, and step execution. Only OAwMCI and CIOA were included for the second step analysis because most of these participants took the $2^{\text {nd }}$ step at the intensity I (OAwMCI, $n=13$; CIOA, $n=9$ ), and all of the participants in these groups took a second compensatory step at intensity II and III. Because very few young adults took a second compensatory step across the intensities, their responses were excluded from the analysis. Post-hoc analysis with Bonferroni's correction was performed to resolve the main effects and interactions (Group $\times$ Intensity). Furthermore, oneway ANOVA was conducted to determine differences within each group and intensity in postural COM state stability, step length, step initiation, and step execution, and was followed up with post-hoc Bonferroni's test to resolve main effects.

For a planned sub-analysis, a $2 \times 2$ repeated measure ANOVA was conducted to compare postural COM state stability at the first compensatory step touch down with the second step at the highest perturbation intensity (intensity III). Post-hoc analysis with Bonferroni's correction was performed to resolve for main effects and interaction (Group $\times$ Intensity).

\section{RESULTS}

\section{Demographics}

The one-way ANOVA between the three groups revealed that there was a significant difference in age $[F(2,42)=138.188, P<0.001]$, however, there was no significant differences in height $[F(2,42)=0.532, P=0.591]$ and weight $[F(2,42)=2.445, P=0.099]$. Furthermore, there was no gender differences $\left[X^{2}(2, N=45)=1.26, P=0.533\right]$ between the three groups. Lastly, one-way ANOVA between CIOA and OAwMCI revealed no significant differences in age 
a)
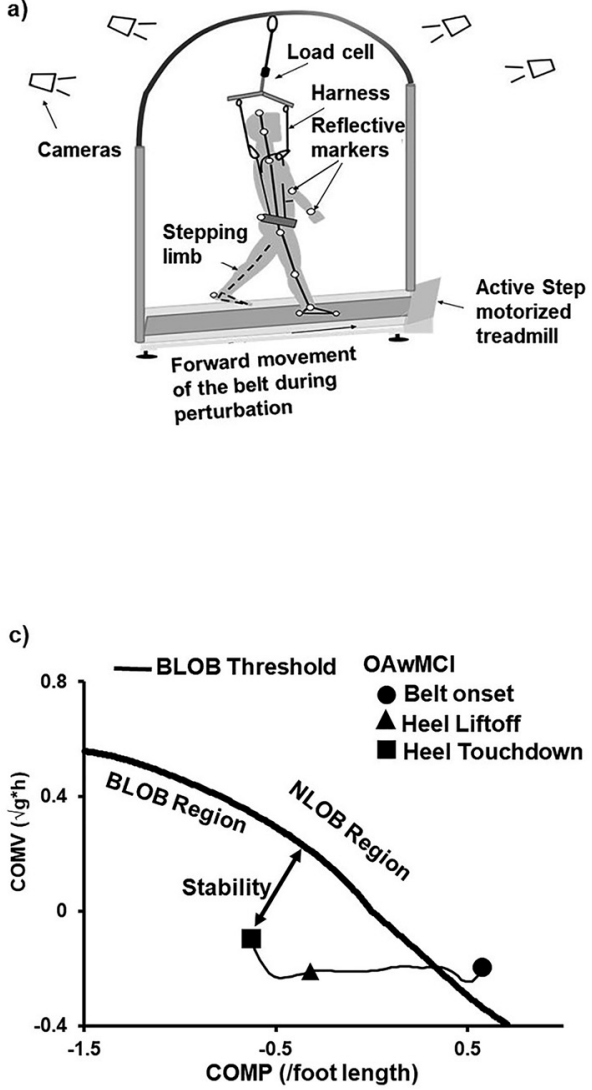

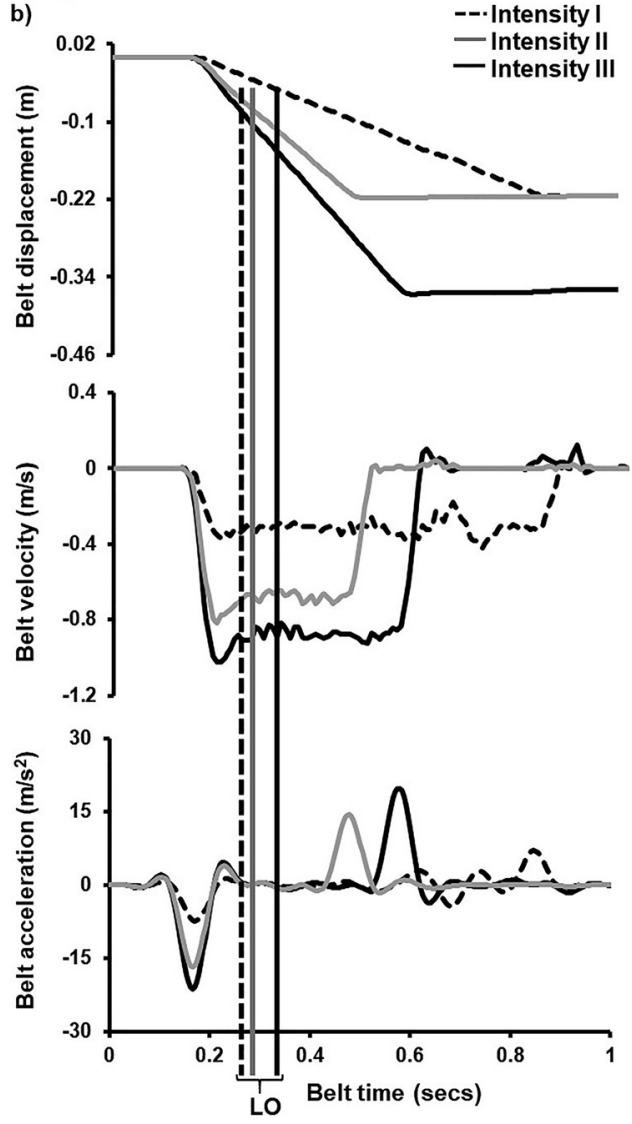

Fig. 1. (a) Experimental setup to of participants exposed to slip-like perturbations in stance. This figure has been adopted from previously published paper [19]. (b) Perturbation-specific information displaying belt displacement, velocity, and acceleration profiles of three perturbation intensities (I, II, \& III). For intensity I, the belt moved forward (slip-like) with a displacement of $0.2 \mathrm{~m}$ for $0.65 \mathrm{~s}$ at $0.31 \mathrm{~m} \mathrm{~s}^{-1}$ with an acceleration of $7.75 \mathrm{~m} \mathrm{~s}^{-2}$. For intensity II, the belt moved forward with a displacement of $0.2 \mathrm{~m}$ for $0.28 \mathrm{~s}$ at

$0.67 \mathrm{~m} \mathrm{~s}^{-1}$ with an acceleration of $16.75 \mathrm{~m} \mathrm{~s}^{-2}$. For intensity III, the belt moved forward with a displacement of $0.3 \mathrm{~m}$ for $0.38 \mathrm{~s}$ at $0.86 \mathrm{~m} \mathrm{~s}^{-1}$ with an acceleration of $21.5 \mathrm{~m} \mathrm{~s}^{-2}$. The vertical lines indicate the compensatory heel liftoff (LO) of an older adult with mild cognitive impairment. (c) The normalized relative center of mass (COM) position (COMP) and velocity (COMV) trace in the anterior-posterior direction of an older adult with mild cognitive impairment (OAwMCI) during a perturbation intensity III plotted against the theoretical boundary for backward balance loss (solid black line) [33]. The trace is shown from onset of belt displacement (closed circle) to post-slip compensatory heel touchdown (closed square). Also shown is the post-slip compensatory step lift off (closed triangle). The absolute COMP is expressed relative to base of support and normalized to foot length. The absolute COMV is expressed relative to base of support and normalized to square root of gravity (g) and body height $(\mathrm{h})$. A negative COMP indicates that it is posterior to the heel and vice versa. A negative COMV indicates that the COM is moving at a slower horizontal velocity than the slipping heel (base of support). The stability value (two headed arrow) represents the shortest distance from an instantaneous COM state (i.e., its position and velocity) to the backward balance loss boundary 
Table 1. Demographics and clinical characteristics of young adults and older adults with and without mild cognitive impairment. BBS $=$ Berg Balance Scale, MoCA $=$ Montreal Cognitive Assessment

\begin{tabular}{|c|c|c|c|c|c|}
\hline & OAwMCI & CIOA & $\begin{array}{l}\text { Young } \\
\text { Adults }\end{array}$ & $\begin{array}{l}\text { Between all } 3 \\
\text { groups } P \\
\text { value }\end{array}$ & $\begin{array}{c}\text { Between } \\
\text { OAwMCI } \\
\text { and CIOA } P \text { value }\end{array}$ \\
\hline Age [Means (SD)] & $66.26(8.72)$ & $\begin{array}{l}61.13 \\
(7.41)\end{array}$ & $\begin{array}{c}26.2 \\
(4.87)\end{array}$ & $<0.001$ & 0.093 \\
\hline Range in years & $56-86$ & $57-71$ & $19-35$ & & \\
\hline Gender $(\mathrm{M} / \mathrm{F})$ & $7 / 8$ & $8 / 7$ & $5 / 10$ & 0.533 & 0.715 \\
\hline $\begin{array}{l}\text { Height }(\mathrm{cm}) \\
\quad[\text { Means }(\mathrm{SD})]\end{array}$ & $169.99(11.74)$ & $\begin{array}{l}171.57 \\
(11.74)\end{array}$ & $\begin{array}{l}167.38 \\
(10.12)\end{array}$ & 0.099 & 0.377 \\
\hline Range in $\mathrm{cm}$ & $144.78-181.61$ & $153-187$ & $\begin{array}{c}185.42- \\
152.4\end{array}$ & & \\
\hline $\begin{array}{l}\text { Weight (lbs) } \\
\quad[\text { Means (SD)] }\end{array}$ & $158.73(27.06)$ & $\begin{array}{c}171.6 \\
(48.43)\end{array}$ & $\begin{array}{l}142.62 \\
(28.31)\end{array}$ & 0.591 & 0.715 \\
\hline Range in lbs & $116-204.2$ & $104-270$ & $95-185$ & & \\
\hline $\begin{array}{l}\text { BBS Out of } 56 \\
{[\text { Means (SD)] }}\end{array}$ & $53.5(2.02)$ & $\begin{array}{l}53.86 \\
(2.19)\end{array}$ & - & - & 0.799 \\
\hline Range & $49-56$ & $48-56$ & & & \\
\hline $\begin{array}{c}\text { MoCA Out of } 30 \\
\text { [Means (SD)] }\end{array}$ & $21.86(2.06)$ & $\begin{array}{l}29.06 \\
(0.79)\end{array}$ & - & - & $<0.001$ \\
\hline Range & $19-25$ & $28-30$ & & & \\
\hline
\end{tabular}

$[F(1,29)=3.016, P=0.093]$, height $[F(1,29)=0.136, P=0.715]$, and weight $[F(1,29)=0.807$, $P=0.377]$. Detailed characteristics are presented in Table 1 .

\section{Perturbation outcome}

Participants in all three groups (OAwMCI, CIOA, and young adults) took a backward compensatory step. Fall percentage during perturbation intensity I among OAwMCI was 20\%, while no falls were observed among CIOA and young adults. At perturbation intensity II, falls among OAwMCI was $26.67 \%$ and $6.67 \%$ among CIOA with no falls observed among young adults. Lastly, at perturbation intensity III, falls among OAwMCI was $66.67 \%$ and $33.33 \%$ among CIOA with no falls among young adults. The $3 \times 3$ repeated measure ANOVA for number of compensatory steps revealed a significant main effect of intensity $[F(2,84)=20.5, P$ $<0.001]$ and main effect of group $[F(2,42)=22.93, P<0.001]$, however, no intensity by group interaction $[F(4,84)=1.69, P=0.15]$ was observed. Post-hoc analyses revealed that the number of compensatory steps was significantly higher for the OAwMCI group compared to CIOA $(P=0.003)$ and young adults $(P<0.001)$, and the number of compensatory steps was significantly higher in CIOA compared to young adults $(P<0.01)$ across all intensities (See Table 2).

\section{Postural COM state stability}

For the first compensatory step, the $3 \times 3$ repeated measure ANOVA revealed a significant main effect of intensity $[F(2,84)=30.513, P<0.001]$, main effect of group $[F(2,42)=42.992$, 
Table 2. Means (standard deviations) of total number of compensatory steps across all intensities between older adults with mild cognitive impairment (OAwMCI), cognitively intact older adults (CIOA), and young adults (YA)

\begin{tabular}{lcccc}
\hline Intensity & OAwMCI & CIOA & Young & Individual comparisons \\
\hline I & $2.33(0.072)$ & $1.66(0.48)$ & $1.2(0.4)$ & OAwMCI $>$ CIOA $^{* *}$ OAwMCI $>Y^{* * *}$ \\
II & $3(0.92)$ & $2.26(0.45)$ & $1.26(0.4)$ & OAwMCI $>$ CIOA* OAwMCI $>$ YA $^{* * *}$ \\
III & $3.13(1.3)$ & $2.33(0.61)$ & $1.7(0.4)$ & OAwMCI $>$ CIOA $>$ YA*** \\
\hline
\end{tabular}

*** $P<0.001 ;{ }^{* *} P<0.01 ;{ }^{*} P<0.05$.

$P<0.001]$, and intensity by group interaction $[F(4,84)=7.529, P<0.001]$. Post-hoc analyses revealed that the postural COM state stability was significantly lower for the OAwMCI group compared to CIOA $(P=0.001)$ and young adults $(P<0.001)$. In addition, the postural COM state stability was significantly lower in CIOA compared to young adults $(P<0.001)$ (Fig. 2a). Additionally, postural COM state stability at perturbation intensity III was significantly lower compared to I and II $(P<0.001)$. Resolving for main effects and interaction, one-way ANOVA results revealed that OAwMCI exhibited significantly deteriorated postural COM state stability compared to their healthy counterparts (Table 3 ).

For the second compensatory step, the $3 \times 2$ repeated measure ANOVA revealed a significant main effect of group $[F(1,20)=9.772, P=0.005]$ but no main effect of intensity $[F(2,40)$ $=2.743, P=0.076]$ and intensity by group interaction $[F(2,40)=1.925, P=0.113]$. Resolving for main effects, one-way ANOVA showed that the postural COM state stability was significantly lower for OAwMCI group compared to CIOA at intensity II $[F(1,21)=6.721, P=$ 0.015] and intensity III $[F(1,20)=9.196, P=0.005]$ (Fig. 2b).

For our planned sub-analysis comparing postural COM state stability between first and second compensatory steps at intensity III, the $2 \times 2$ repeated measure ANOVA revealed a significant main effect of step $[F(1,28)=22.668, P<0.001]$ and main effect of group $[F(1,28)$ $=13.617, P<0.001]$ but no step by group interaction $[F(1,28)=1.289, P=0.266]$. Post-hoc analysis showed significant difference between first and second compensatory steps $(P=0.001)$.

Step length. For the first compensatory step, the $3 \times 3$ repeated measure ANOVA revealed a significant main effect of intensity $[F(2,84)=22.714, P<0.001]$, main effect of group $[F(2,42)$ $=19.631, P<0.001]$, and intensity by group interaction $[F(4,84)=6.341, P<0.001]$. Post-hoc analyses revealed that compared to young adults, the step length was significantly lower for the CIOA $(P<0.001)$ and OAwMCI group $(P<0.001)$ (Fig. $2 \mathrm{c})$. Resolving for main effects and interaction, one-way ANOVA results revealed that OAwMCI exhibited significantly reduced step length compared to their healthy counterparts (Table 3 ).

For the second compensatory step, the $3 \times 2$ repeated measure ANOVA did not reveal significant main effect of group $[F(1,20)=0.340, P=0.56]$, main effect of intensity $[F(2,40)=$ $2.861, P=0.069]$, or intensity by group interaction $[F(2,40)=1.030, P=0.366]$ (Fig. $2 \mathrm{~d}$ ).

Step initiation. For the first compensatory step, the $3 \times 3$ repeated measure ANOVA revealed a significant main effect of group $[F(2,42)=8.093, P=0.001]$ but no main effect of intensity [ $F$ $(2,84)=0.202, P=0.818]$ or intensity by group interaction $[F(4,84)=0.899, P=0.469]$. Post- 


\section{OYoung OCIOA OOAWMI}

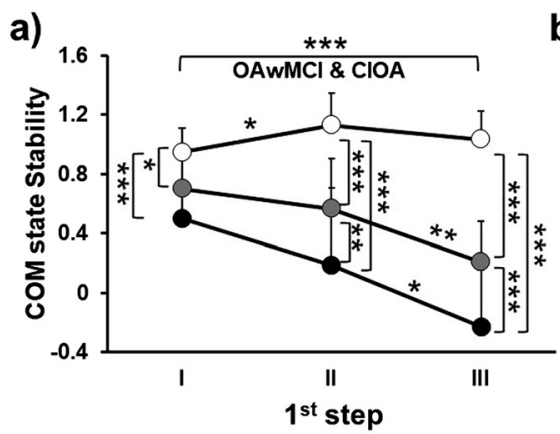

b)

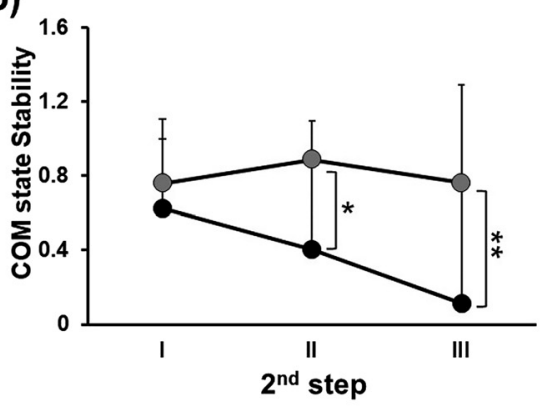

c)

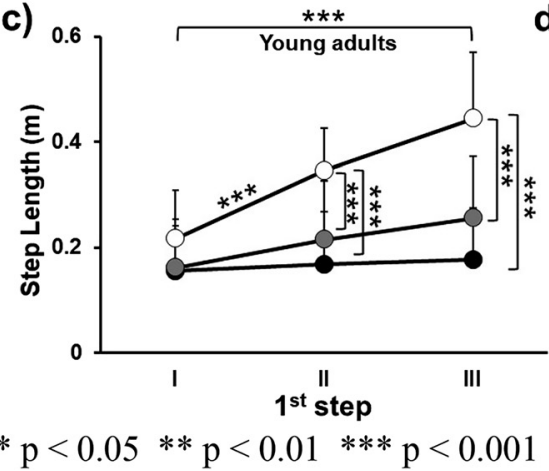

$* \mathrm{p}<0.05 * * \mathrm{p}<0.01 * * * \mathrm{p}<0.001$

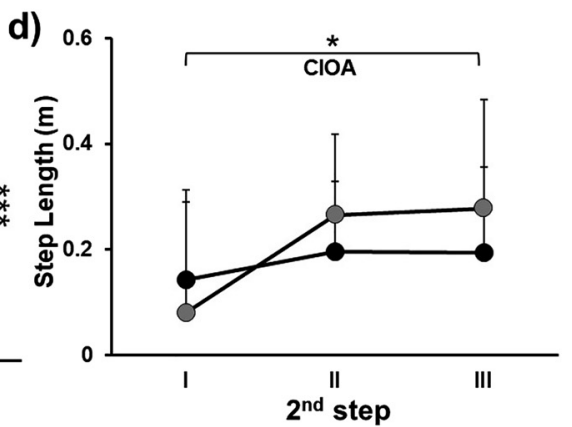

Fig. 2. Means and SD of COM state stability with a significant group and intensity differences at first (a) and second (b) compensatory step heel touch down in response to increasing slip-like perturbation intensities. The COM state stability was the lowest among older adults with mild cognitive impairment

(OAwMCI) compared to cognitively intact older adults (CIOA) and young adults during the first compensatory step. Of the people in CIOA and OAwMCI groups who took a second compensatory step, COM state stability persisted to be significantly lower among the OAwMCI group at intensities II and III. Means and SD of step length (in meters, $\mathrm{m}$ ) with a significant group and intensity differences at first (c) and second (d) compensatory step heel touch down in response to increasing slip-like perturbation intensities. The step length was the lowest among older adults with mild cognitive impairment (OAwMCI) compared to cognitively intact older adults (CIOA) and young adults during the first compensatory step. Furthermore, the second compensatory step length was lower among the OAwMCI group compared to CIOA, but the results were not statistically significant. It should be noted that while CIOA significantly increased (scaled) their step length at intensity III compared to intensity II, OAwMCI shows similar performance irrespective of the perturbation intensity

hoc analyses revealed that compared to young adults $(P=0.001)$ and CIOA $(P=0.034)$, the step initiation time was significantly higher in OAwMCI group $(P \leq 0.01)$ (Fig. 3a). Resolving for main effect results, one-way ANOVA results revealed that OAwMCI exhibited significantly greater step initiation time compared to their healthy counterparts (Table 3). 
Table 3. One-way ANOVA results for first compensatory step among older adults with mild cognitive impairment (OAwMCI), cognitively intact older adults (CIOA), young adults (YA) across intensities I (L1), II (L2) and III (L3)

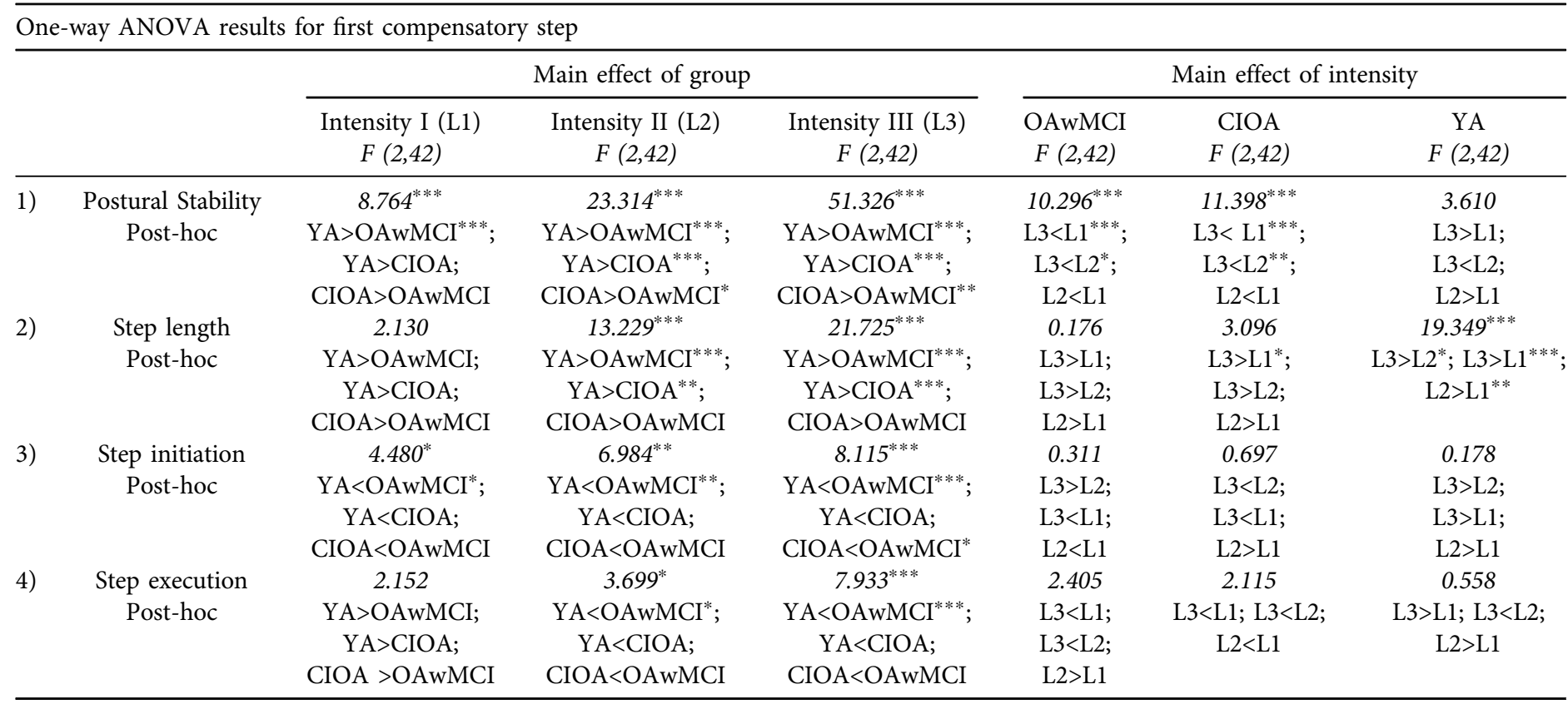

\footnotetext{
${ }^{* * *} P<0.001 ;{ }^{* *} P<0.01 ;{ }^{*} P<0.05$.
} 


\section{OYoung OCIOA O OAWMCI}
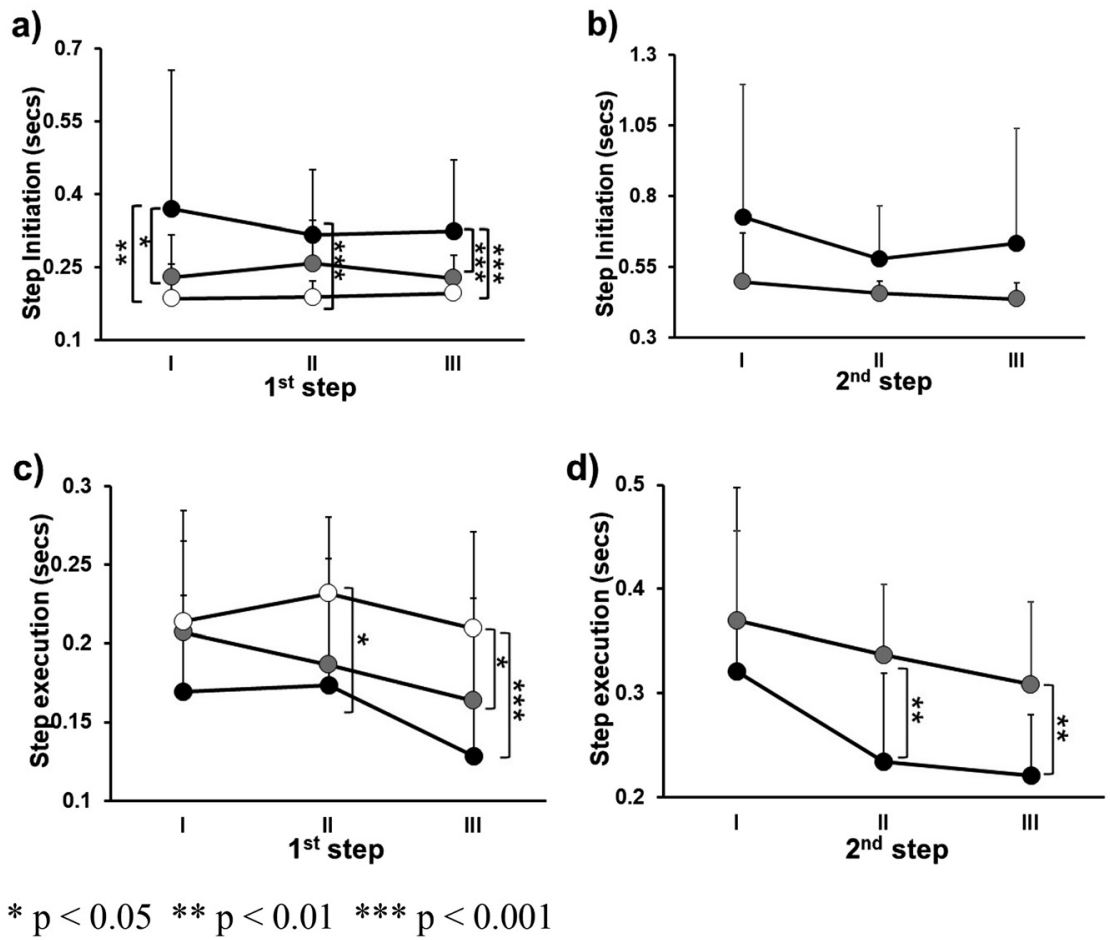

Fig. 3. Means and SD of step initiation (measured in seconds) with a significant group differences at first (a) and second (b) compensatory step heel touch down in response to increasing slip-like perturbation intensities. The step initiation was the highest (i.e., took a long time to initiate a response) among older adults with mild cognitive impairment (OAwMCI) compared to cognitively intact older adults (CIOA) and young adults during the first compensatory step. A similar trend was observed for the second compensatory step among the OAwMCI group compared to CIOA, but the results were not statistically significant. Means and SD of step execution (measured in seconds) with a significant group differences at first (c) and second (d) compensatory step heel touch down in response to increasing slip-like perturbation intensities. The step execution was the lowest among older adults with mild cognitive impairment (OAwMCI) compared to cognitively intact older adults (CIOA) and young adults during the first compensatory step. A similar trend was observed for the second compensatory step among the OAwMCI group compared to CIOA which was

significant at II and III intensities indicating that they needed multiple steps to re-establish stability

For the second compensatory step, the $3 \times 2$ repeated measure ANOVA did not reveal significant main effect of intensity $[F(2,40)=1.557, P=0.223]$ or main effect of group [ $F$ ( 1 , $20)=3.177, P=0.090]$ or intensity by group interaction $[F(2,40)=0.259, P=0.773]$ (Fig. 3b).

Step execution. For the first compensatory step, the $3 \times 3$ repeated measure ANOVA revealed a significant main effect of intensity $[F(2,84)=5.543, P=0.005]$ and main effect of group [ $F(2$, $42)=6.933, P=0.003]$ but no intensity by group interaction $[F(4,84)=1.057, P=0.383]$. 
Post-hoc analyses revealed that compared to young adults, the step execution time was significantly lower in OAwMCI group $(P=0.002)$, however, did not significantly differ from CIOA $(P$ $=0.261)$. Additionally, CIOA did not significantly differ from young adults $(P=0.167)$ (Fig. 3c). Resolving for main effect results, one-way ANOVA results revealed that OAwMCI exhibited significantly reduced step execution time compared to their healthy counterparts (Table 3).

For the second compensatory step, the $3 \times 2$ repeated measure ANOVA revealed a significant main effect of group $[F(1,20)=5.006, P=0.011]$ and main effect of intensity $[F(2,40)=$ 5.006, $P=0.011]$ but no significant intensity by interaction $[F(2,40)=0.897, P=0.416]$. Resolving for main effects, one-way ANOVA showed that step execution time was significantly lower in OAwMCI group compared to CIOA at intensity II $(F(1,21)=13.271, P=0.001)$ and intensity III $[F(1,21)=11.923, P=0.002]$ but not at intensity $\operatorname{I}[F(1,21)=0.576, P=0.457]$

(Fig. 3d).

\section{DISCUSSION}

Our study aimed to examine reactive balance control in older adults with mild cognitive impairment (OAwMCI) compared to their healthy counterparts and further investigate their ability to scale balance recovery responses against increasing slip-like stance perturbation intensities. The results showed that unlike young adults and cognitively intact older adults (CIOA), OAwMCI exhibited deteriorated reactive balance control (i.e., showed decreased postural COM state stability) and were unable to scale their postural COM state stability as the perturbation intensity altered. Additionally, compared to young adults, OAwMCI and CIOA exhibited deteriorated compensatory step characteristics (i.e., reduced step length, increased step initiation time, and reduced step execution time), especially at the highest perturbation intensities (intensity III).

Firstly, marked differences among the groups in all the reactive balance control measures were observed, demonstrating the inability of OAwMCI to initiate a rapid, effective compensatory step to re-establish stability regardless of the type of perturbation intensity. Although all participants experienced a backward loss of balance following a slip-like stance perturbation (indicated by the need to take a compensatory posterior step), OAwMCI exhibited significantly reduced postural COM state stability at intensities II and III compared to young adults and CIOA for the first compensatory step (Fig. 2a). It is known that postural COM state stability during dynamic tasks is influenced by center of mass (COM) position and velocity relative to the base of support (BOS) [33]. Previous studies have indicated that during a stance perturbation, COM position has a greater influence on stability than velocity, with COM position at touchdown of the first compensatory step modulated primarily by its length. A large compensatory step not only extends the BOS and maximizes the distance between COM position and BOS, but could simultaneously help decelerate the COM velocity to regain the perturbation induced instability and recover balance [36, 9]. The step length (spatial parameter) between OAwMCI and CIOA did not significantly differ at any perturbation intensity; however, both groups were significantly lower compared to young adults at intensity II and III (Fig. 2c). The decreased postural COM state stability at the first compensatory step touch down in CIOA and OAwMCI probably resulting from a reduced step length compared to the young, could have led to procure 
multiple steps to recover balance loss (See Table 2). On the other hand, temporal parameter (i.e., step initiation time) was significantly higher among OAwMCI compared to CIOA and young adults at intensity I and III. This may indicate that while aging significantly affects spatial parameters regardless of cognitive state, additional cognitive decline may significantly affect temporal parameters more.

Secondly, our results demonstrated that unlike the young adults and CIOA, OAwMCI were unable to modulate their postural COM state stability based on the perturbation intensity. It is previously shown that participants increase or decrease the magnitude of their postural responses [37], compensatory step length [19], and/or postural COM state stability [19] based on the intensity (displacement/velocity/acceleration) induced [19]. Our study results are consistent with these previous findings, where the ability to modulate balance recovery responses (in this case step length and postural stability) was demonstrated maximally by the young adults [19]. Our results indicate that OAwMCI had more difficulty in modulating postural COM state stability with increasing intensity than CIOA; although the latter also showed difficulty in scaling their postural stability on intensity II and III compared to younger adults on the first compensatory step. This was suggested by the fact that compared to no difference at intensity I between CIOA and OAwMCI, there was a significant difference at intensity II and III in postural COM state stability for the first compensatory step between the two groups. Furthermore, CIOA took fewer compensatory steps compared to OAwMCI on intensities II and III, and for $2^{\text {nd }}$ compensatory step, CIOA had greater postural COM state stability and were able to scale their responses (no difference between intensity II and intensity III) (Fig. 2a and b). Although the first compensatory step length showed significant scaling in young adults, both OAwMCI and CIOA did not show significant scaling in step length for the $1^{\text {st }}$ compensatory step. However, a positive trend of scaling step length was observed among CIOA compared to OAwMCI at intensity II and III for the $1^{\text {st }}$ and $2^{\text {nd }}$ compensatory step. While step length is a significant contributor to modulate postural COM state stability to stance perturbations, there may be other factors that could have affected this. Such probability could explain why significant scaling was seen in postural COM state stability but not in step length. Furthermore, the reduced step length in OAwMCI than in CIOA on the first compensatory step could have resulted in OAwMCI taking more steps ( $>2$ steps) than CIOA, especially at the higher intensity.

This is the first study to our knowledge to investigate the effect of cognitive impairment on reactive balance control by exposure to large-scale support surface perturbations. It is suggested that the significantly deteriorated reactive balance control demonstrated in OAwMCI could result from impaired sensory stimuli detection (i.e., interpret perturbation specific information such as displacement/velocity/acceleration), and/or disrupted sensory integration and/or motor execution. Such proposition is supported by and could be attributed to the significant pathological changes within the cortical and subcortical areas (e.g., gray matter atrophy, white matter abnormalities) in OAwMCI which could affect the neurophysiological processes involved in the control of reactive balance $[25,12,26,27]$. Furthermore, previous studies have reported that OAwMCI exhibit disrupted sensory integration that adversely impacts balance control and gait performances $[38,39]$. Thus, the delayed step initiation may indicate the inability of OAwMCI to relay and interpret perturbation-specific information (displacement/velocity/acceleration), thereby delaying information processing. Such delay combined with errors in selection and modulation of the appropriate motor response based on the estimated COM state would 
ultimately be affecting the execution of response, thus resulting in a shorter length of the compensatory step and lower postural COM state stability.

Based on our study findings, deteriorated reactive balance responses and the inability to scale their responses against different perturbation intensities among CIOA and OAwMCI indicates the inability to process the perturbation-specific signal by higher-cortical areas due to ageassociated decline with or without additional cognitive impairment. It is postulated that based on prior experience, the CNS has the ability to make online corrections of balance recovery responses based on the perturbation-specific sensory information perceived [40]. All participants received a familiarization trial before the actual test, which may have been enough to create a "prior experience" context before they randomly received perturbations at different intensities. While young adults were able to appropriately modulate (enhance postural COM state stability with an increase in compensatory step length) their response from intensity I to intensity II and maintain this for intensity III, both CIOA and OAwMCI were unable to do the same and showed a decline in postural COM state stability with increasing intensities. The responses of CIOA may be attributed to age-associated decline, and further deteriorated performance of OAwMCI may be accredited to changes in cortical morphology and a lack of modulation from the same $[25,12,26,27]$. This is supported by the fact that CIOA was able to recover their postural COM state stability and demonstrate no decline in it with increasing perturbation intensity on the $2^{\text {nd }}$ compensatory step. However, OAwMCI were unable to significantly change performance by their $2^{\text {nd }}$ compensatory step with postural COM state stability remaining inferior to CIOA at heel touch down. Such results further strengthen the postulation that even a mild decline in the cognitive state could significantly affect the reactive balance control mechanism necessary for fall recovery.

Few studies have recently shown that about 50\% of OAwMCI demonstrate vestibular dysfunction resulting in impaired spatial awareness (self-motion perception, navigation, memory) associated with balance control [41-44]. While the primary afferent pathway for balance control and organization of rapid recovery responses is the sensory system - visual, somatosensory, and vestibular - the reliance on one component increases when any other is conflicted. The inability to decipher sensory information (displacement/velocity/acceleration) increases the reliability of the vestibular system which is often impaired in OAwMCI [19,44]. This could have further inhibited the ability to interpret sensory information and modulate postural responses based on prior experience as the perturbation intensity altered.

\section{Limitations of the study}

The postulations made from our study are preliminary and reactive responses among OAwMCI is still at its infancy. Despite the study results, our study has certain limitations. Firstly, the sample size in our study for each group $(n=15)$ is low which may result in smaller effect size and higher sample may yield better and concrete understanding of scaling balance recovery responses in OAwMCI compared to CIOA. Secondly, our study was limited to examining biomechanical variables and did not evaluate neuromuscular responses of balance recovery against altering perturbation intensities. Future studies examining neuromuscular responses via electromyographical recordings could provide further mechanistic insight into the impairments observed. Furthermore, given that this is the first study to determine reactive responses in OAwMCI, future studies with a larger sample size need to further validate the results. The extent 
to which subjective cognitive decline affects other biomechanical determinants (trunk control, hip height) of falls still needs to be explored in this population and may help validate the findings of our study. Thirdly, our study did not specifically assess for vestibular dysfunction, thus future studies may focus on determining whether such association could mediate reactive balance control responses in OAwMCI. Lastly, future studies may incorporate examination of reactive balance control responses among different types of mild cognitive impairment (amnestic and non-amnestic). Considering this was the first study of its kind, the differentiation of types of mild cognitive impairment was not included.

\section{CONCLUSION}

This exploratory study provides preliminary findings that significant cognitive decline compared to normal aging could influence reactive balance responses against large magnitude perturbations and affects modulating these responses based on the perturbation intensities. Furthermore, our results suggest that while aging does affect scaling of balance recovery responses, cognitive decline in older adults may affect such modulation as perturbation intensity increases, thus contributing to exacerbation of instability in OAwMCI and predisposing them to higher fall-risk compared to their healthy counterparts. However, the results provide only preliminary understanding of reactive balance responses in OAwMCI and more research is required to determine whether such impaired responses are primarily due to cognitive decline.

\section{ACKNOWLEDGMENTS}

We thank the funding sources of the study which was funded in part by a pilot grant awarded to Dr. Tanvi Bhatt by the Midwest Roybal Center for Health Promotion and Translation (NIH/ NIA ADRD supplemental grant 2P30AG022849-11). We also would like to thank Shuaijie Wang and Gonzalo Varas for their contribution to data collection without whom this study would not have been successful.

\section{REFERENCES}

1. Petersen RC, Smith GE, Waring SC, Ivnik RJ, Kokmen E, Tangelos EG. Aging, memory, and mild cognitive impairment. Int Psychogeriatr 1997; 9(S1): 65-9.

2. Plassman BL, Langa KM, Fisher GG, Heeringa SG, Weir DR, Ofstedal MB, et al. Prevalence of cognitive impairment without dementia in the United States. Ann Intern Med 2008; 148(6): 427-34.

3. Villemagne VL, Burnham S, Bourgeat P, Brown B, Ellis KA, Salvado O, et al. Amyloid $\beta$ deposition, neurodegeneration, and cognitive decline in sporadic Alzheimer's disease: a prospective cohort study. Lancet Neurol 2013; 12(4): 357-67.

4. Kallin K, Gustafson Y, Sandman P-O, Karlsson S. Factors associated with falls among older, cognitively impaired people in geriatric care settings: a population-based study. Am J Geriatr Psychiatry 2005; 13(6): 501-9. 
5. Shaw FE. Falls in cognitive impairment and dementia. Clin Geriatr Med 2002; 18(2): 159-73.

6. Bárrios H, Narciso S, Guerreiro M, Maroco J, Logsdon R, de Mendonça A. Quality of life in patients with mild cognitive impairment. Aging Ment Health 2013; 17(3): 287-92.

7. Doi T, Shimada H, Makizako H, Tsutsumimoto K, Hotta R, Nakakubo S, et al. Mild cognitive impairment, slow gait, and risk of disability: a prospective study. J Am Med Directors Assoc 2015; 16(12): $1082-6$.

8. Petersen RC. Mild cognitive impairment as a diagnostic entity. J Intern Med 2004; 256(3): $183-94$.

9. Maki BE, McIlroy WE. The role of limb movements in maintaining upright stance: the "change-in-support" strategy. Phys Ther 1997; 77(5): 488-507.

10. Pai Y-C, Bhatt TS. Repeated-slip training: an emerging paradigm for prevention of slip-related falls among older adults. Phys Ther 2007; 87(11): 1478-91.

11. Pieruccini-Faria F, Sarquis-Adamson Y, Montero-Odasso M. Mild cognitive impairment affects obstacle negotiation in older adults: results from "Gait and Brain Study". Gerontology 2019; 65(2): 164-73.

12. Leandri M, Cammisuli S, Cammarata S, Baratto L, Campbell J, Simonini M, et al. Balance features in Alzheimer's disease and amnestic mild cognitive impairment. J Alzheimer's Dis 2009; 16(1): 113-20.

13. Shin BM, Han SJ, Jung JH, Kim JE, Fregni F. Effect of mild cognitive impairment on balance. J Neurol Sci 2011; 305(1-2): 121-5.

14. Mansfield A, Peters AL, Liu BA, Maki BE. Effect of a perturbation-based balance training program on compensatory stepping and grasping reactions in older adults: a randomized controlled trial. Phys Ther 2010; 90(4): 476-91.

15. Tangen GG, Engedal K, Bergland A, Moger TA, Mengshoel AM. Relationships between balance and cognition in patients with subjective cognitive impairment, mild cognitive impairment, and Alzheimer disease. Phys Ther 2014; 94(8): 1123-34.

16. Burleigh A, Horak F. Influence of instruction, prediction, and afferent sensory information on the postural organization of step initiation. J Neurophysiol 1996; 75(4): 1619-28.

17. Timmann D, Horak F. Perturbed step initiation in cerebellar subjects 1 . Modifications of postural responses. Exp Brain Res 1998; 119(1): 73-84.

18. Timmann D, Horak F. Perturbed step initiation in cerebellar subjects: 2. Modification of anticipatory postural adjustments. Exp Brain Res 2001; 141(1): 110-20.

19. Patel PJ, Bhatt T. Does aging with a cortical lesion increase fall-risk: examining effect of age versus stroke on intensity modulation of reactive balance responses from slip-like perturbations. Neuroscience 2016; 333: 252-63.

20. Lin S-I, Woollacott MH. Postural muscle responses following changing balance threats in young, stable older, and unstable older adults. J Mot Behav 2002; 34(1): 37-44.

21. Moreland JD, Richardson JA, Goldsmith CH, Clase CM. Muscle weakness and falls in older adults: a systematic review and meta-analysis. J Am Geriatr Soc 2004; 52(7): 1121-9.

22. Bastian AJ. Understanding sensorimotor adaptation and learning for rehabilitation. Curr Opin Neurol 2008; 21(6): 628.

23. Wang Y, Wang S, Lee A, Pai Y-C, Bhatt T. Treadmill-gait slip training in community-dwelling older adults: mechanisms of immediate adaptation for a progressive ascending-mixed-intensity protocol. Exp Brain Res 2019; 237(9): 2305-17.

24. Thompson JD, Plummer P, Franz JR. Age and falls history effects on antagonist leg muscle coactivation during walking with balance perturbations. Clin Biomech 2018; 59: 94-100.

25. Dickerson B, Salat D, Greve D, Chua E, Rand-Giovannetti E, Rentz D, et al. Increased hippocampal activation in mild cognitive impairment compared to normal aging and AD. Neurology 2005; 65(3): 404-11. 
26. Wang L, Goldstein FC, Veledar E, Levey AI, Lah JJ, Meltzer CC, et al. Alterations in cortical thickness and white matter integrity in mild cognitive impairment measured by whole-brain cortical thickness mapping and diffusion tensor imaging. Am J Neuroradiology 2009; 30(5): 893-9.

27. Zhang Y, Schuff N, Camacho M, Chao LL, Fletcher TP, Yaffe K, et al. MRI markers for mild cognitive impairment: comparisons between white matter integrity and gray matter volume measurements. PloS One 2013; 8(6): e66367.

28. Nasreddine ZS, Phillips NA, Bédirian V, Charbonneau S, Whitehead V, Collin I, et al. The Montreal Cognitive Assessment, MoCA: a brief screening tool for mild cognitive impairment. J Am Geriatr Soc 2005; 53(4): 695-9.

29. Hans D, Schott A, Meunier P. Ultrasonic assessment of bone: a review. The Eur J Med 1993; 2(3): 157-63.

30. Zagzebski JA, Rossman PJ, Mesina C, Mazess RB, Madsen EL. Ultrasound transmission measurements through the os calcis. Calcified Tissue Int 1991; 49(2): 107-11.

31. Lajoie Y, Gallagher S. Predicting falls within the elderly community: comparison of postural sway, reaction time, the Berg balance scale and the Activities-specific Balance Confidence (ABC) scale for comparing fallers and non-fallers. Arch Gerontol Geriatr 2004; 38(1): 11-26.

32. Yang F, Bhatt T, Pai Y-C. Role of stability and limb support in recovery against a fall following a novel slip induced in different daily activities. J Biomech 2009; 42(12): 1903-8.

33. Pai Y-C, Iqbal K. Simulated movement termination for balance recovery: can movement strategies be sought to maintain stability in the presence of slipping or forced sliding? J Biomech 1999; 32(8): 779-86.

34. McMahon TA. Muscles, reflexes, and locomotion. Princeton University Press; 1984.

35. Smith JA, Fisher BE. Anticipatory postural adjustments and spatial organization of motor cortex: evidence of adaptive compensations in healthy older adults. J Neurophysiol 2018; 120(6): 2796-805.

36. Hsiao-Wecksler ET. Biomechanical and age-related differences in balance recovery using the tether-release method. J Electromyogr Kinesiol 2008; 18(2): 179-87.

37. Welch TD, Ting LH. A feedback model explains the differential scaling of human postural responses to perturbation acceleration and velocity. J Neurophysiol 2009; 101(6): 3294-309.

38. Mahoney JR, Wang C, Dumas K, Holtzer R. Visual-somatosensory integration in aging: does stimulus location really matter? Vis Neurosci 2014; 31(3): 275.

39. Mahoney JR, Verghese J. Does cognitive impairment influence visual-somatosensory integration and mobility in older adults? J Gerontol Ser A 2020; 75(3): 581-8.

40. Jacobs J, Horak F. Cortical control of postural responses. J Neural Transm 2007; 114(10): 1339.

41. Brandt T, Schautzer F, Hamilton DA, Brüning R, Markowitsch HJ, Kalla R, et al. Vestibular loss causes hippocampal atrophy and impaired spatial memory in humans. Brain 2005; 128(11): 2732-41.

42. Hufner K, Stephan T, Hamilton D, Kalla R, Glasauer S, Strupp M, et al. Gray-matter atrophy after chronic complete unilateral vestibular deafferentation. Ann New York Acad Sci 2009; 1164(1): 383-5.

43. Micarelli A, Viziano A, Della-Morte D, Augimeri I, Alessandrini M. Degree of functional impairment associated with vestibular hypofunction among older adults with cognitive decline. Otol Neurotol 2018; 39(5): e392-400.

44. Ventre-Dominey J. Vestibular function in the temporal and parietal cortex: distinct velocity and inertial processing pathways. Front Integr Neurosci 2014; 8: 53. 